\title{
Effect of microwave heating on the regeneration of modified activated carbons saturated with phenol
}

\author{
B. S. Ondon • B. Sun - Z. Y. Yan • \\ X. M. Zhu • H. liu
}

Received: 17 June 2013/Accepted: 17 December 2013/Published online: 10 January 2014

(C) The Author(s) 2014. This article is published with open access at Springerlink.com

\begin{abstract}
The purpose of this work was to investigate the effect of microwave irradiation on the regeneration of modified activated carbons (GAC/MW, GAC/Ni, and $\mathrm{GAC} / \mathrm{Cu}$ ). The untreated activated carbon (GAC pure) was used for blank experiment. Microwave heating was used for preparation and regeneration of the modified activated carbons. The effect of loading $\mathrm{Ni}^{2+}$ and $\mathrm{Cu}^{2+}$ ions on the activated carbon adsorption capacity was investigated. The results showed that the activated carbon loaded with $\mathrm{Ni}^{2+}$ has no significant effect on phenol adsorption, while the adsorption capacity of activated carbon loaded with $\mathrm{Cu}^{2+}$ significantly decreased. Microwave irradiation showed a positive effect on activated carbon adsorption capacity. Under optimal conditions, the results showed that there was no effect when changing temperature and $\mathrm{pH}$. The effect of ions $\mathrm{Ni}^{2+}$ and $\mathrm{Cu}^{2+}$ loaded into activated carbon were also investigated. During the regeneration process, the activated carbon loaded with $\mathrm{Ni}^{2+}$ showed a strong microwave energy adsorption than the activated carbon loaded with $\mathrm{Cu}^{2+}$. The effect increasing $\mathrm{Ni}^{2+}$ quantity decreases the activated carbon regeneration efficiency. During the regeneration of activated carbons, the highest temperature was observed in the cases of GAC/Ni. During regeneration, the temperature increases when the quantity $\mathrm{Ni}^{2+}$ loaded increases. The regeneration efficiency of activated carbons reaches $98 \%$ even after 10 times of regeneration cycles. After several regenerations, MW/GAC and GAC/Ni regeneration efficiency was high, while regeneration
\end{abstract}

B. S. Ondon $(\varangle) \cdot$ B. Sun $(\varangle) \cdot$ Z. Y. Yan · X. M. Zhu · H. liu College of Environmental Science and Engineering, Dalian Maritime University, Dalian 116026, China

e-mail: brimondondoctor@gmail.com

B. Sun

e-mail:sunb88@dlmu.edu.cn efficiency of $\mathrm{GAC} / \mathrm{Cu}$ decreased considerably. GAC regeneration efficiency also decreased several cycles. During regeneration process, phenol was simply desorbed from activated carbons under microwave irradiation.

Keywords Phenol - Microwave energy ·

Modified activated carbons - Microwave regeneration ·

Microwave irradiation

\section{Introduction}

The discharge of wastewater represents one of the major industrial pollutions (Nabarlatz et al. 2012). Among the hazardous organic wastes produced by the industries, phenolic compounds are the most common organic compound produced (Bo et al. 2008). Phenols are dangerous organic pollutants because they are toxic even at low concentrations (Ahmaruzzaman and Sharma 2005). These phenolic compounds usually have a low threshold in potable waters and also have a detrimental effect on human health at high level (Hui et al. 2011). One of the most important and more efficient techniques used to adsorb or retain the contamination (Manh et al. 2011) is adsorption. Among the materials commonly used as adsorbent, activated carbons is one of the most used materials (Lei et al. 2011) due to their high pore. Adsorption by activated carbons (AC) is a well-established technology widely used in wastewater purification (Bandosz 2006; Campbell et al. 2011).

Regeneration is time and energy consuming and very often there is a significant deterioration of the adsorbent's pore structure ( $\mathrm{Lu}$ et al. 2011), thereby reducing the final adsorption capacity and the efficiency of the regeneration (Ania et al. 2004). In recent years, microwave (MW)

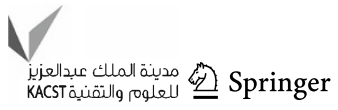


irradiation has attracted attention as a tool for regeneration due to its capability of molecular-level heating. Interesting reports have appeared on the application of MW-heating technology for regenerating AC (Price and Schmidt 1997; Bradshaw et al. 1997; Coss and Cha 2000; Liu et al. 2004; Jones et al. 2002; Deming et al. 2011; Guido et al. 2008; Bermudez et al. 2012) with very promising results. Applications of microwave regeneration technologies are lagely used in industries for economical purposes (Remya and Lin 2011). Microwave regeneration offers potential advantages over conventional treatment (Wang et al. 2012) and catalytic reactions (Huan et al. 2010). This technology can also be used for catalyst preparation (Xinliang et al. 2012). Researches on regeneration of the activated carbon loaded with metal are less reported in literature. To the best of our knowledge, loading metal on activated carbon decreases activated carbon adsorption capacity (Ania et al. 2007). In this work, microwave irradiation has a positive effect on modified activated carbon regeneration increasing their adsorption capacity after cycles of regenerations (Yuen and Hameed 2009). These investigations are done to evaluate the effect of microwave regeneration on the activated carbons loaded with metals. The effect of microwave irradiation may have many effects on the modified activated carbons. On the other hand, the effect of the nature and quantity of metal loaded upon activated carbons decreases activated carbon regeneration efficiency. So far interaction may occur between the metal and the activated carbon.

\section{Experimental section}

\section{Reagents and instruments}

A charcoal granulated activated carbon (GAC) with particle size of $0.1-1.1 \mathrm{~mm}, \mathrm{Cl}(\%)=0.025$ and $\mathrm{Pb}$ $(\%)=0.005, \mathrm{Fe}(\%)=0.02 \mathrm{pH}(50 \mathrm{~g} / \mathrm{l}, 25 \mathrm{C})=5.0-70$, $\mathrm{Zn}(\%)=0.05$ was purchased from Tianjin Weichen chemical reagent. Phenol solid from Shenyang chemical industry $0.1 \mathrm{~g}$ was diluted in distillated water to make a $1,000 \mathrm{ppm}$ solution and diluted to obtain the solution at different concentrations. $\mathrm{Cu}\left(\mathrm{NO}_{3}\right) \cdot \mathrm{H}_{2} \mathrm{O}$ and $\mathrm{Ni}\left(\mathrm{NO}_{3}\right)$ $\mathrm{H}_{2} \mathrm{O}$ were purchased from Tianjin chemical industry China. The electronic scale balance (JJ 1,000, Max. 1,000 g. High-performance liquid Chromatography (HPLC) (LC-10UV, equipped with UV detector (UV-1575) and C18 reverse-phase column $(250 \times 4.6 \mathrm{~mm}, 5$ m ODS, China) was used for the analyses. Magnetic agitator was used to stir the phenol solution and also to set the temperature of the solutions. $\mathrm{pH}$ meter PHs-3C (Shang Hai, China) was used for $\mathrm{pH}$ measures.

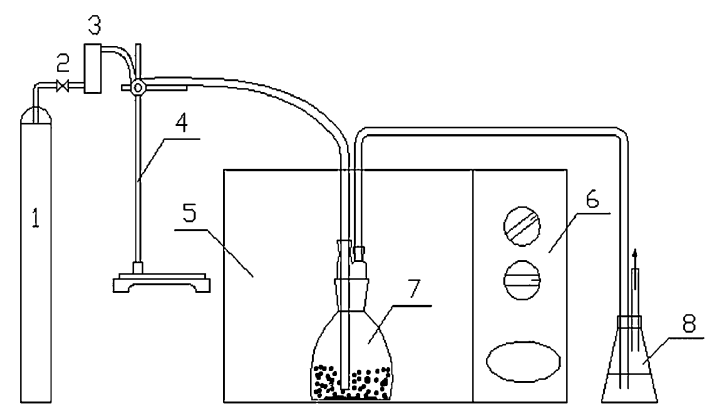

Fig. 1 Schematic of the apparatus used for the preparation and regeneration of the modified activated carbons 1 nitrogen gas container, 2 bottle opener, 3 flow pressure meter, 4 support, 5 microwave oven, 6 timer, 7 conical quartz glass, 8 water container glass

\section{Experimental apparatus of the modified domestic microwave oven}

The experimental device is a modified domestic microwave oven which operates at 2,540 MHz. The microwave oven was modified such as to introduce nitrogen into the conical quartz glass. Nitrogen gas bottle was connected with the flow pressure meter (Fig. 1). The conical quartz glass was used as reactor for each experiments. The modified activated carbons prepared were cooled at room temperature under the protection of nitrogen gas and were removed from the microwave oven to be used for the next experiments.

\section{Preparation of the modified activated carbons}

The impregnation was done by microwave calcination. The solutions were prepared as follow: $3.78 \mathrm{~g}$ of $\mathrm{Cu}$ $\left(\mathrm{NO}_{3}\right) \cdot \mathrm{H}_{2} \mathrm{O}, 5.01 \mathrm{~g}$ of $\mathrm{Ni}\left(\mathrm{NO}_{3}\right) \mathrm{H}_{2} \mathrm{O}$ were introduced into two different conical flask, $50 \mathrm{ml}$ of deionized water was added to make copper nitrate and nickel nitrate solutions, then $20 \mathrm{~g}$ of granular activated carbons (GAC) was introduced into the solutions. Then, the mixture was introduced in the machine for rotation at the speed of $232 \mathrm{rev} / \mathrm{min}$ during $2.5 \mathrm{~h}$. The weight percentages of metals are, $\mathrm{Cu}: \mathrm{GAC}=1: 20$ and Ni:GAC $=1: 20$. The mixed solutions were introduced in the quartz conical flask reactor separately and placed in the microwave oven. The microwave irradiation power was $400 \mathrm{~W}$ and the solutions were treated under the same conditions: intermittently irradiated (for $1 \mathrm{~min} 46 \mathrm{~s}, 2 \mathrm{~min}$, and $50 \mathrm{~s}$ ) until nearly dryness. The Nitrogen gas was introduced at the pressure flow of $0.4 \mathrm{~m}^{3} / \mathrm{h}$ during 2 min before irradiation and was kept 5 min during the irradiation time at $400 \mathrm{~W}$. 


\section{Saturation of the activated carbons}

In each experiment, $2 \mathrm{~g}$ of activated carbons was used in $250 \mathrm{ml}$ conical flash glass and $200 \mathrm{ml}$ of phenol solution was introduced in the glass and stirred for $2 \mathrm{~h}$ until the activated carbons reached the adsorption equilibrium. $0.05 \mathrm{ml}$ was taken each time to be analyzed. The concentration and removal efficiency of phenol were calculated following the Eqs. (1) and (2).

$c=c_{\mathrm{o}} \times \frac{A}{A_{\mathrm{o}}}$

$q=100 \% \times\left(1-\frac{c}{c_{\mathrm{o}}}\right)$

$C$ and $C$ o are, respectively, phenol solution concentration and phenol initial concentration $(\mathrm{mg} / \mathrm{l}) . A$ and $A o$ are, respectively, peak surface of phenol solution and peak surface of phenol initial solution $(\mathrm{uV} \times \mathrm{s}) . q$ is the percentage of phenol removal by the activated carbon (\%).

\section{Regeneration of modified activated carbons}

Experiments were conducted in the conical quartz glass of $250 \mathrm{ml}$ (Fig. 1). The experimental apparatus used to regenerate the activated carbons is shown in the figure (Fig. 1). After regeneration, the activated carbons were according to the optimized adsorption conditions. Under these conditions, the regeneration of activated carbons was done several times from 1 to 10 cycles of regeneration after several adsorptions. The regeneration of the activated carbons was done under a flow of nitrogen gas (Fig. 1) to avoid destruction of activated carbons. In our previous studies, we investigated the optimal condition for microwave regeneration of the modified activated carbon (Brim et al. 2013). The activated carbons were regenerated according to the optimized conditions.

\section{Regeneration conditions}

Microwave irradiation power and time have been optimized. After a series of regeneration experiments, MW power of $700 \mathrm{~W}$ and $3 \mathrm{~min}$ of regeneration time were selected as the optimized conditions. Under nitrogen atmosphere pressure, flow $\mathrm{N}_{2}$ flow is $0.4 \mathrm{~m}^{3} / \mathrm{h}$. The gas was first introduced for $1 \mathrm{~min}$ before irradiation and during 3 min of irradiation time.

\section{Analytical methods}

Phenol was analyzed by a high-performance liquid, the detection wavelength was set at $270 \mathrm{~nm}$, and the mobile
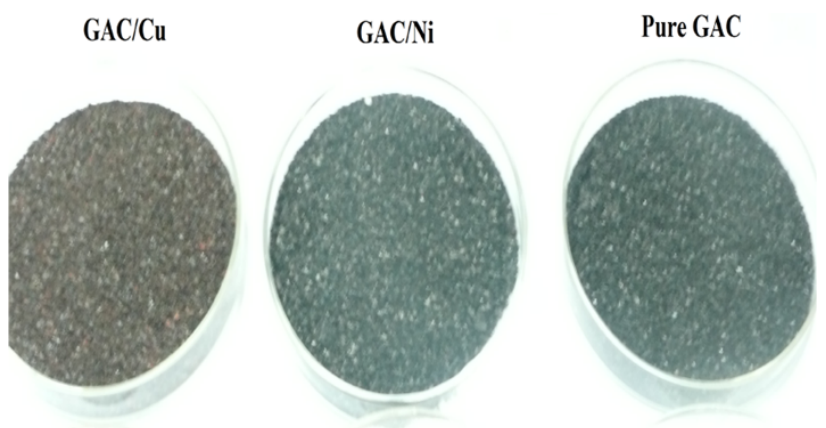

Fig. 2 Photograph of the microwave prepared modified activated carbons

phase was 40:60 (v/v) acetonitrile and water at a flow rate of $0.8 \mathrm{ml} \mathrm{min}^{-1}$ flow rate. $0.05 \mathrm{ml}$ was taken each time to be analyzed. And the injection volume was $20 \mu \mathrm{l}$.

\section{Results and discussion}

Photograph-modified activated carbons

The photograph of the as-prepared modified activated carbon is represented. As we can see in the figure below (Fig. 2), the different activated carbons are presented with different color.

The activated carbon loaded with nickel as shown in the middle of this picture is colored in blue (Fig. 2). But the activated carbon loaded with copper is represented in a red brown color and as to make the comparison the activated carbon simply treated with microwave irradiation is colored in the black with the same color as that of the untreated activated carbon. These results shows that the color in the activated carbons loaded with copper and nickel are not the consequence of microwave irradiation but by the fact of loading metal in the activated carbon micro pores and each color, respectively, represents the nature of metal loaded inside of the activated carbon micro pores. The as-prepared colored activated carbons were directly used as they appear for next experiences.

\section{Influence of regeneration parameters on activated carbon regeneration efficiency}

Influence of regeneration time

Regeneration of activated carbons is a very crucial problem (Ania et al. 2004). Many investigations have been done to optimize the conditions for high regeneration efficiency of activated carbons. In this study, optimal condition for microwave regeneration of different types of modified activated carbons was investigated.GAC/Ni and $\mathrm{GAC} / \mathrm{Cu}$

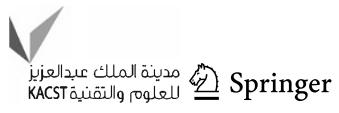




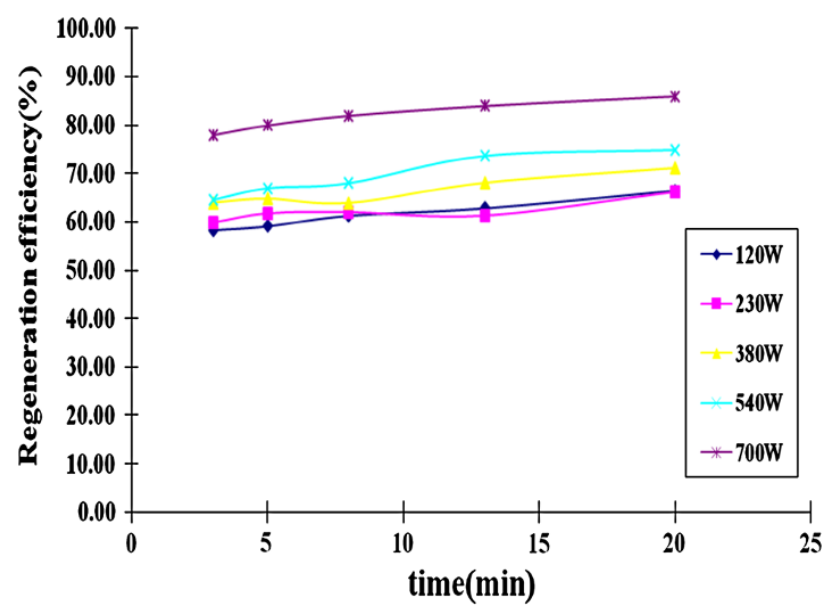

Fig. 3 Influence of time on the AC regeneration efficiency

were chosen as the modified activated carbon. (Fig. 2). The regeneration efficiency depends relatively on the microwave irradiation time (Fig. 3). The highest regeneration efficiency is obtained when obviously the irradiation power was set at $700 \mathrm{~W}$. At lowest microwave irradiation power, there is not significant increase of regeneration efficiency (Fig. 3). The selected time was, respectively, 3, 5, 8, 13 and $20 \mathrm{~min}$. The activated carbon regeneration efficiency increases with the irradiation time (Fig. 3).

\section{Influence of microwave regeneration power}

As it can be seen in the figure below, when the microwave irradiation power is high, activated carbon regeneration efficiency is also high (Fig. 4). Activated carbon removal efficiency reaches 80-90\% at the maximum microwave irradiation of $700 \mathrm{~W}$. The more activated carbons is exposed

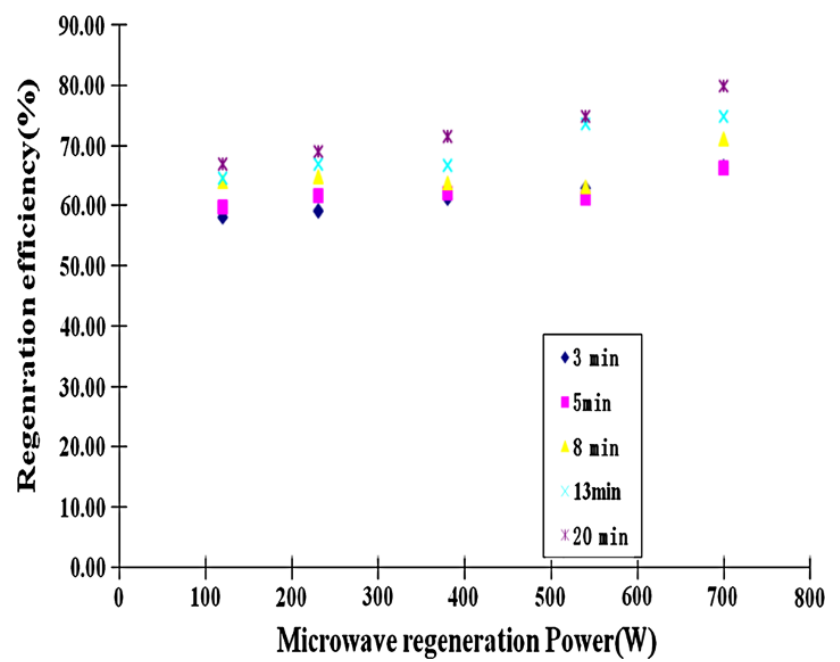

Fig. 4 Influence of microwave power on the $\mathrm{AC}$ regeneration efficiency under MW irradiation, the high is the regeneration efficiency (Fig. 4). The highest regeneration efficiency was obtained at the microwave irradiation power of $700 \mathrm{~W}$ and $20 \mathrm{~min}$ of irradiation time. From 3 to $20 \mathrm{~min}$, the difference of regeneration efficiency is not significant (Fig. 4). This slight difference may be explained by the fact that at $5 \mathrm{~min}$, phenol compounds adsorbed by activated carbons were almost desorbed. For simple reasons of energy consumption, the time of regeneration was $3 \mathrm{~min}$ and the microwave irradiation power was set at $700 \mathrm{~W}$ for next experience unless otherwise mentioned. Three minutes and $700 \mathrm{~W}$ of microwave power were selected as the optimized regeneration conditions of the modified activated carbon regeneration. MW power was thus seated at $700 \mathrm{~W}$ and the regeneration time was $3 \mathrm{~min}$.

\section{Comparison of the effect of loading $\mathrm{Ni}^{2+}$ and $\mathrm{Cu}^{2+}$ on the Activated Carbons regeneration efficiency}

The regeneration efficiency of $\mathrm{GAC} / \mathrm{Ni}$ and $\mathrm{GAC} / \mathrm{Cu}$ was investigated under similar conditions. The results were analyzed in comparison with the untreated activated carbon (pure GAC).The activated carbon impregnated with nickel presented the highest regeneration efficiency (Fig. 5). The result remains identical from 5 to $20 \mathrm{~min}$. This result shows that the regeneration efficiency of $\mathrm{GAC} / \mathrm{Ni}$ is higher than $\mathrm{GAC} / \mathrm{Cu}$. The effect of microwave heating on the regeneration efficiency of GAC/Ni is more efficient than GAC/ $\mathrm{Cu}$. This result can be explained by the fact that $\mathrm{Ni}$ adsorbs more microwave irradiation than $\mathrm{Cu}$. The difference of regeneration efficiency of $\mathrm{GAC} / \mathrm{Ni}$ and $\mathrm{GAC} / \mathrm{Cu}$ is very significant. Microwave energy has a positive effect on the activated carbon impregnated with nickel than the activated carbon impregnated with copper (Fig. 5).

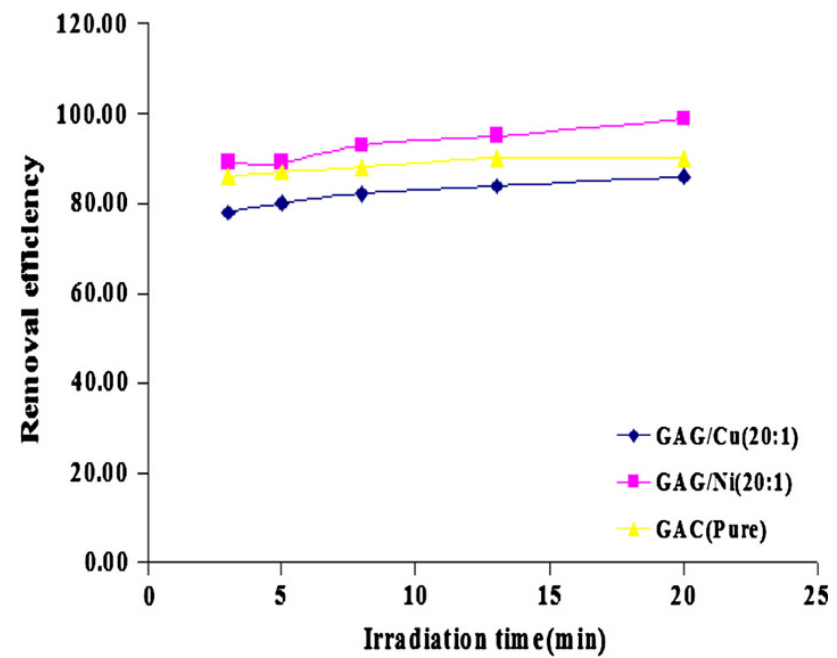

Fig. 5 Comparison of the effect loading $\mathrm{Ni}$ and $\mathrm{Cu}$ on the regeneration efficiency 


\section{Evolution of activated carbon temperature upon regeneration cycles}

Temperature is a very important parameter in the regeneration of activated carbons (Ania et al. 2004). The evolution of temperature was controlled after each cycle of regeneration. Temperature plays an important role on activated carbon regeneration efficiency (J.M. et al. 2011). Carbons are used for dyes adsorption. The previous results showed that regeneration efficiency increases with the activated carbon temperature (Calis and Bermudez 2012). The activated carbons loading with nickel has the highest regeneration efficiency. The modified activated carbon $(\mathrm{GAC} / \mathrm{Ni})$ seems to absorb microwave energy better than $\mathrm{GAC} / \mathrm{Cu}$ and this adsorption of microwave energy is the principal cause of elevation temperature in the GAC/Ni system. The temperature of activated carbons can reach $400^{\circ}$ in the case of $\mathrm{GAC} / \mathrm{Ni}$, while it is below $400^{\circ}$ in the case of $\mathrm{GAC}$ and $\mathrm{GAC} / \mathrm{Cu}$ (Fig. 5). GAC/Cu adsorbs less microwave energy. These results can explain the low temperature observed in the activated carbon loaded with $\mathrm{Cu}^{2+}$ while activated carbons were regenerated (Fig. 6). The regeneration temperature of $\mathrm{GAC} / \mathrm{Cu}$ is around $300^{\circ}$, while the regeneration temperature of $\mathrm{GAC} / \mathrm{Ni}$ is around $450^{\circ}$ (Fig. 6). These results show that nickel can absorb microwave energy more than copper while the activated carbons are being regenerated. On the other hand, GAC treated with microwave energy absorbs better microwave energy than the as-received GAC. The activated carbon pretreated with microwave energy (MW/GAC) seems to be able to absorb more microwave irradiation than the asreceived activated carbon (GAC) (Fig. 6). The temperature of the modified activated carbons decreases with the number of regeneration cycle. When the modified activated carbons are regenerated, their temperature decreases

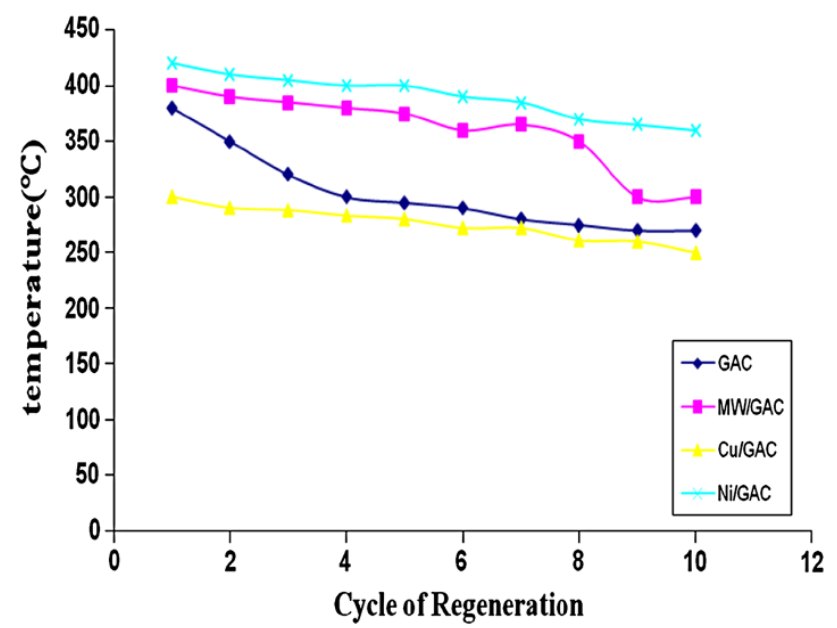

Fig. 6 Evolutions of activated carbons temperature function of number regeneration cycle slightly (Fig. 6). More the activated carbons regeneration time is extented, less the activated carbons temperature is high.

\section{Comparison of regeneration efficiency of activated carbons after several cycles}

The different activated carbons could be regenerated with the percentage of regeneration reaching $94 \%$ after 10 cycles of regeneration (Fig. 7). The microwave pretreated GAC and GAC/Ni have high adsorption capacity before the activated carbons are regenerated. After ten regeneration cycles the adsorption capacity of $\mathrm{GAC} / \mathrm{Cu}$ is very low comparatively to GAC/Ni as (Fig. 7). Compared to $\mathrm{GAC}$ and $\mathrm{GAC} / \mathrm{Ni}$, the regeneration efficiency of GAC/ $\mathrm{Cu}$ was very low (Fig. 7). The regeneration efficiency of activated carbon untreated GAC remained almost constant after several cycle of regeneration as shown in Fig. 6. This effect could be attributed to the action of microwave irradiation that acts to the GAC pore volume and pore size to increase the volume pore of the activated carbons (Bandosz 2006). Microwave irradiation has the effect on the activated carbon surface chemistry, thus increasing the activated carbon adsorption capacity. Obviously, when metal is uploaded into the activated carbon pore, it decreases their adsorption capacity. The results obtained in this study prove that the effect of MW irradiation on GAC seems to be more dominant (Bathen 2003) (Fig. 7).

After several regeneration cycles, the adsorptive capacity of $\mathrm{GAC} / \mathrm{Cu}$ decreases considerably while $\mathrm{GAC} / \mathrm{Ni}$ remained high during ten regeneration cycles as shown in Fig. 7. This fact could be attributed to the fact that Ni (II)

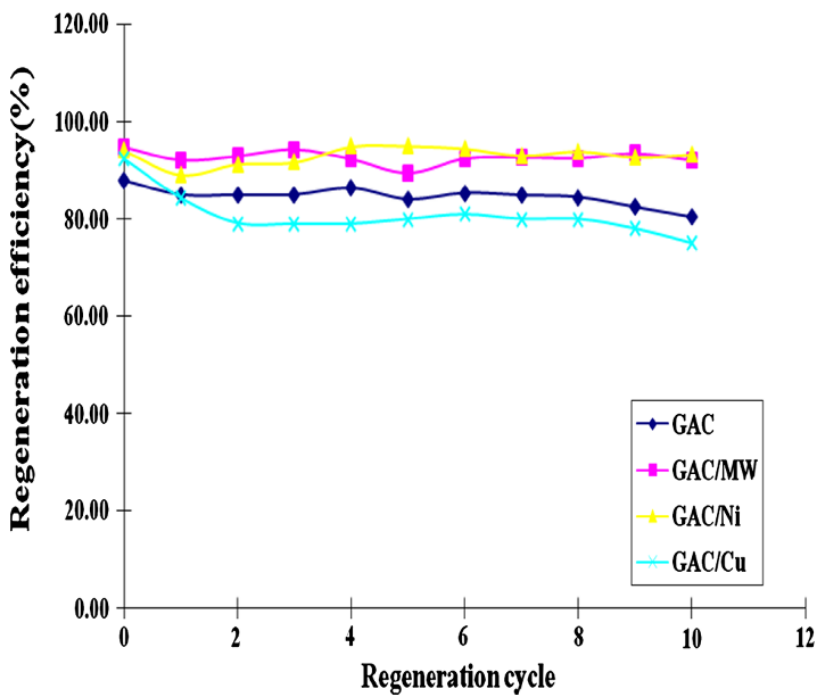

Fig. 7 Comparison of the adsorptive capacities of different activated carbon after 10 cycles of regenerations 
adsorbs more MW irradiation, while $\mathrm{Cu}$ (II) has a negative effect on microwave irradiation or has more interaction with the activated carbon surface chemistry thus reducing the activated carbon adsorption capacity in aqueous solution. The adsorptive capacity of the as-received activated carbon (GAC) is high comparatively to $\mathrm{GAC} / \mathrm{Cu}$ (Fig. 7). This result can be explained by the fact that the adsorption capacity of the activated carbon loaded with copper (GAC/ $\mathrm{Cu})$ decreases when the activated carbon is regenerated several times under microwave irradiation. These results confirm that the activated carbon loaded with $\mathrm{Cu}$ (II) does not adsorb sufficient microwave irradiations allowing the elevation of temperature meaning that $\mathrm{Cu}$ (II) adsorbs less or almost not the microwave irradiations allowing the decrease of GAC/Cu adsorptive capacity (Fig. 7).

\section{Influence of nickel quantity on activated carbon regeneration cycles}

The effect of the quantity of Ni loaded on activated carbon was also investigated when activated carbons were regenerated. To understand the effect of metal nickel loaded on activated carbon, the GAC/Ni (20:1) and GAC/Ni (10:1) were regenerated under similar conditions. The results show that the more nickel metal is loaded on activated carbon, the regeneration efficiency decreases significantly (Fig. 8). Obviously, because the activated carbon pores are saturated with $\mathrm{Ni}$, the activated carbon adsorption capacity decreases (Fig. 8).It has been published that loading $\mathrm{Ag}^{+}$, $\mathrm{Ni}^{2+}, \mathrm{Cu}^{2+}$ or $\mathrm{Zn}^{2+}$ ion on the activated carbon improves activated carbon adsorption capacity upon some

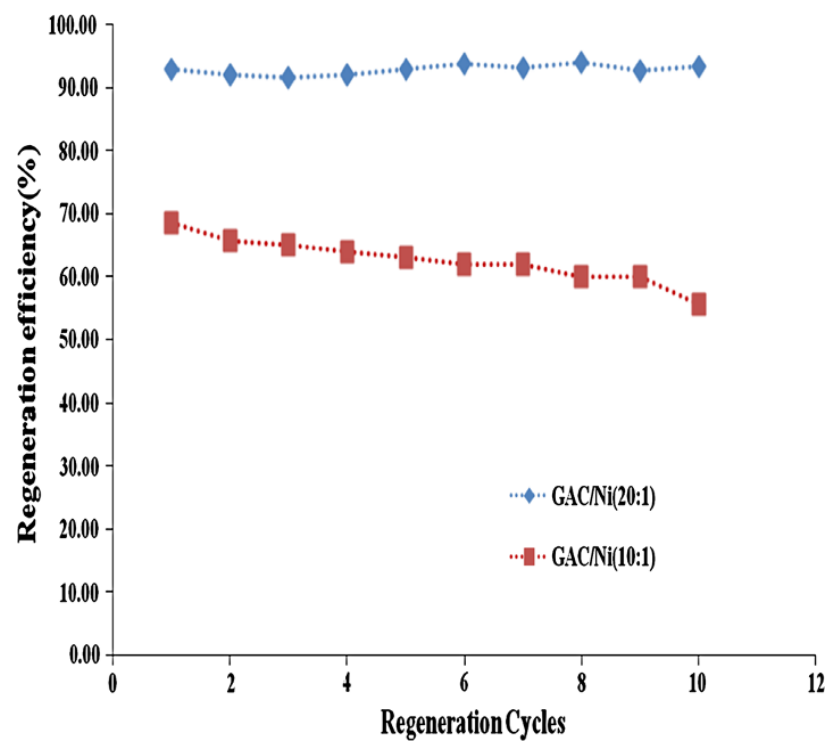

Fig. 8 Influence of nickel quantity upon regeneration efficiency after 10 cycles of regeneration compounds like benzothiophene (Moxin et al. 2006; Loredana and Mihael 2012). During our experiments the activated carbon loaded with $\mathrm{Ni}^{2+}$ higher adsorption capacity upon phenol adsorption but when the $\mathrm{Ni}^{2+}$ increased, the activated carbon adsorption capacity considerably decreased. The activated carbon regeneration efficiency decreases with nickel quantity and upon the several regeneration cycles.

\section{Conclusion}

Under the optimum conditions, phenol was absorbed by the modified activated carbons. The modified activated carbons were saturated with phenol. After the maximum saturation, the modified activated carbons were regenerated by microwave irradiation under the protection of nitrogen gas. The activated carbon loaded with $\mathrm{Cu}^{2+}$ presented a considerable decrease upon phenol adsorption, while the activated carbon loaded with $\mathrm{Ni}^{2+}$ did not decrease upon phenol adsorption. The optimum conditions of the activated carbon regeneration were found. Under a microwave irradiation power of $700 \mathrm{~W}$ and $3 \mathrm{~min}$ of irradiation time, the regeneration efficiency of the modified activated carbons could reach around $98 \%$.

The activated carbons could regenerate with the regeneration efficiency of $98 \%$ after 10 regenerations cycles.GAC/MW, GAC/Ni and $\mathrm{GAC} / \mathrm{Cu}$ adsorptive capacity were higher than GAC before regeneration. The regeneration efficiency of $\mathrm{GAC} / \mathrm{Ni}$ and $\mathrm{GAC} / \mathrm{MW}$ remained high after 10 regenerations cycles, while $\mathrm{GAC} /$ $\mathrm{Cu}$ decreased significantly. This proves that Ni (II) plays an important role on the regeneration process compared with $\mathrm{Cu}$ (II). The regeneration efficiency of the activated carbon loaded with $\mathrm{Ni}^{2+}$ is higher than the activated carbon loaded with $\mathrm{Cu}^{2+}$. Activated carbon loaded with $\mathrm{Ni}^{2+}$ can absorb more microwave energy than the one loaded with $\mathrm{Cu}^{2+}$. During the regeneration process, the temperature of the modified activated carbons decreases with cycles of regenerations. The activated carbon temperature was measured. The regeneration temperature of $\mathrm{GAC} / \mathrm{Ni}$ was higher than $\mathrm{GAC} / \mathrm{Cu}$, GAC/MW and untreated GAC over several cycles of regenerations. The quantity of metal $\mathrm{Ni}^{2+}$ loaded into activated carbon significantly decreases activated carbon regeneration efficiency. Even after several regeneration cycles, the regeneration efficiency remained decreased.

Acknowledgments We thank the support of the National Natural Science Foundation (NSFC-10875019, 41005079). This research is supported by "The Fundamental Research Funds for the Central Universities". We are pleased to acknowledge the financial support of this study to the Chinese council scholarship, Dalian Maritime University and the Congolese government for their support. 
Open Access This article is distributed under the terms of the Creative Commons Attribution License which permits any use, distribution, and reproduction in any medium, provided the original author(s) and the source are credited.

\section{References}

Ahmaruzzaman M, Sharma DK (2005) Adsorption of phenol from wastewater. J Colloid Interface Sci 278:14-24

Ania CO, Parra JB, Menendez JA, Pis JJ (2004) Microwave induced regeneration of activated carbons polluted with phenol. A comparison with conventional thermal regeneration. Carbon 42:1377-1381

Ania C, Parra J, Menendez A, Pis J (2007) Microwave-assisted regeneration of activated carbons loaded with pharmaceuticals. Water Res 41:3299-3306

Bandosz TJ (2006) Activated carbon surfaces in environmental remediation. Interface science and technology series. Elsevier, New York

Bathen D (2003) Physical waves in adsorption technology: an overview. Sep Purif Technol 33:163-177

Bermudez JM, Fidalgo B, Arenilllas A, Menendez JA (2012) Mixtures of steel-making slag and carbons as catalysts or microwave assisted dry reforming of $\mathrm{CH} 4$. J Chin Catal 23:1115-1118

Bo L, Quan X, Wang X (2008) Preparation and characteristics of carbon-supported platinum catalyst and its application in the removal of phenolic pollutants in aqueous solution by microwave-assisted catalytic oxidation. J Hazard Mater 157:179-186

Bradshaw S, Van J, De Swardt J (1997) Preliminary economic assessment of microwave regeneration of activated carbon for the carbon in pulp process. International Microwave Power Institute. J Microw Power Electromagn Energy 32:131-144

Brim SO, Sun B, Zhiyu Y, Xiaomei Z, Liu H (2013) Investigation of the optimal conditions for microwave regeneration of modified activated carbons saturated with phenol. (ICEES 2013) 978-184626

Calis E, Bermudez JM (2012) Low temperatures regeneration of activated carbons using microwaves: revising conventional wisdom. J Environ Manag 102:134-140

Campbell QP, Bunt JR, Kasaini H, Kruger DJ (2011) The preparation of activated carbon from South African coal. J South Afr Inst Min Metall 112:37-44

Coss M, Cha CY (2000) Microwave regeneration of activated carbon used for removal of solvents from vented air. J Air Waste Manag Assoc 50:529-535

Deming Z, Jie C, Michael R (2011) Kinetics of microwave-enhanced oxidation of phenol by hydrogen peroxide. Environ Sci Eng 5:57-64
Guido B, Silvia B, Carlo R, Laura A (2008) Technologies for the removal of phenol from fluid streams: a short review of recent developments. J Hazard Mater 160:265-288

Huan H, Shaogui Y, Kai Y, Yongming J, Cheng S, Wang Lianhong (2010) Microwave induced catalytic degradation of crystal violet in nano-nickel dioxide suspensions. J Hazard Mater 173:393-400

Hui HT, Chi Y, Lu F, Chang M, Yen W (2011) Catalytic removal of $\mathrm{NO}$ and PAHs over AC-supported catalysts from incineration flue gas: bench-scale and pilot-plant tests. Chem Eng J 169:135-143

Jones DA, Lelyveld TP, Mavrofidis SD, Kingman SW, Miles NJ (2002) Microwave heating applications in environmental engineering-a review. Resour Conserv Recycl 34:75-90

Lei Z, Xinyu Z, Xingjia G, Xiaoyan S, Xueyan L (2011) Investigation on the degradation of acid fuchsin induced oxidation by $\mathrm{MgFe} 2 \mathrm{O} 4$ under microwave irradiation. J Mol Catal A Chem 885:81-87

Liu X, Quan X, Bo L, Chen S, Zhao Y (2004) Simultaneous pentachlorophenol decomposition and granular activated carbon regeneration assisted by microwave irradiation. Carbon 42:415-422

Loredana V, Mihael N (2012) Adsorption isotherms of phenol and aniline on activated carbon. Rev Roum Chim 57:85-93

Lu P, Lin, Lin HC, Yu W, Cherm JM (2011) Chemical regeneration of activated carbons used for dyes adsorption. J Taiwan Inst Chem Eng 42:305-311

Manh HD, Ngoc HP, Thi DN, Thi T (2011) Activated carbon/Fe3O4 nanoparticle composite: fabrication, methyl orange removal and regeneration by hydrogen peroxide. Chemosphere 85:1269-1276

Moxin Y, Zhong L, Qibin X (2006) Effects of different metal ions loaded onto activated carbon on adsorption of benzothiophene. J Chem Ind Eng 57:1943-1948

Nabarlatz D, Celis J, Bonelli P (2012) Bath and dynamic sorption of $\mathrm{Ni}$ (II) ions by activated carbon based on native lignocellulosic precursor. J Environ Manag 95:109-115

Price DW, Schmidt PS (1997) Microwave regeneration of adsorbents at low pressure: experimental kinetics studies. J Microw Power Electromagn Energy 32:145-154

Remya N, Lin JG (2011) Current status of microwave application in wastewater treatment: a review. Chem Eng J 166:797-813

Wang Y, Wang, Xia SQ (2012) In vitro production of phenolic compounds and antioxidant activity in callus suspension cultures of Habenaria edgeworthii: a rare Himalayan medicinal orchid. Ind Crop Products 39:1-218

Xinliang L, Xiaotao T, Chenguang L, Yunqi L (2012) Performance of $\mathrm{Fe} / \mathrm{AC}$ catalyst prepared from demineralized pine bark particles in a microwave reactor. Chem Eng J 193-194:187-195

Yuen FK, Hameed BH (2009) Recent developments in the preparation and regeneration of activated carbons by microwaves. Adv Colloid Interface Sci 149(1-2):19-27 